\title{
The Increasing importance of Normal Flora - Commensals in Health or Potentially Pathogens for Disease
}

We live in a microbial world and our body contains thousands of species of bacteria, protozoa, fungi and even viruses and yet they do not cause any harm in their normal abode. These microorganisms primarily reside in our Skin and mucous membrane which are exposed to the external environment. The normal flora keeps out the pathogen by the virtue of physical occupancy and production of bacteriocins. The definition of virulence and pathogenicity is very elusive as is also the fact that no microorganism is intrinsically benign or pathogenic. Under the right circumstances any microorganism can grow in our body to cause disease. The line of demarcation of normal flora from the pathogenic bacteria are often fuzzy. Members of the normal flora often cause disease when they invade deeper tissues and organs. Consider the Meningococcus or the Pneumococcus. Either one is found in about $10-20 \%$ of the healthy people as normal commensal flora but in remaining $80-90 \%$ of population they can potentially cause morbid diseases. Even the same microbe which existed as normal flora can become pathogenic in the same individual when the host defensive mechanisms are impaired as in the immunocompromised and debilitated individuals. Anaerobic bacteria of the Genus Bacteroides are carried in large numbers in the large intestine and may produce abscesses if they penetrate into deeper tissue via traumatic or Surgical wounds. Occasional outbreaks of disease due to staphylococci, E. coli and other gram negatives coanbe traced to skin, Nose and perianal carriage in health care workers. Infection control measures if strictly followed can keep these type of outbreaks to a minimum level. E. coli and other Gram negatives from the intestines are few well recognized pathogens which act as an endogenous source of sepsis anywhere in the body. Their importance in causing nosocomial infection is well documented. Candida, a harmless commensal becomes a common agent of pneumonia in ICU patients and patients treated with broad spectrum antibioltcs have overgrowth in their bowels of not only Candida but also Clostridium difficile whose toxin can produce severe colitis. In a hospitalized patient Candida can enter the bloodstream via the indwelling intravenous cannula or catheters. Staph. epidermidis can cause infection of the Prosthetic devices in the body. Viridians streptococci enter the bloodstream at the time of dental surgery and can cause sub-acute bacterial endocarditis. Pneumocystis jiroveci, a common inhabitant of the lung is one of the principal cause of death in AIDS patients.

Lactobacilli Keeps the $\mathrm{pH}$ of adult vagina acidic which prevents the establishment of potential pathogens especially during menstruation when the endometrium is raw with open blood vessel through which microbes can invade deeper tissue if they are able to cross the vagina. In infants who are solely milk fed lactobacilli not only prevents the potential pathogens growth but also helps in digestion. This concept has nowadays led to use of probiotics in food. E.coli and other intestinal bacteria synthesize vitamin $\mathrm{k}$. One of the additional but debatable benefits of intestinal bacteria is the constant antigenic stimulation resulting in antibodies production of $\operatorname{Ig} \mathrm{A}$ class which are secreted throughout the mucous membrane and act as a 1 st line of defense.

In a microbe laden world, it is reasonable to conclude that the normal flora is adapted to do more good than harm provided good control measures are adopted at the right time and place.

Prof. Munir Hassan

Prof. M. A. Bashar 